\title{
Effect of dietary fat intake and genetic risk on glucose and insulin-related traits in Brazilian young adults
}

\author{
Sooad Alsulami ${ }^{1,2}$ - Nathália Teixeira Cruvinel ${ }^{3}$. Nara Rubia da Silva • Ana Carolina Antoneli ${ }^{3}$ Julie A. Lovegrove ${ }^{1,4}$. \\ Maria Aderuza Horst ${ }^{3} \cdot$ Karani Santhanakrishnan Vimaleswaran ${ }^{1,4,5}$
}

Received: 10 May 2021 / Accepted: 16 July 2021 / Published online: 13 August 2021

(c) The Author(s) 2021

\begin{abstract}
Purpose The development of metabolic diseases such as type 2 diabetes (T2D) is closely linked to a complex interplay between genetic and dietary factors. The prevalence of abdominal obesity, hyperinsulinemia, dyslipidaemia, and high blood pressure among Brazilian adolescents is increasing and hence, early lifestyle interventions targeting these factors might be an effective strategy to prevent or slow the progression of T2D.

Methods We aimed to assess the interaction between dietary and genetic factors on metabolic disease-related traits in 200 healthy Brazilian young adults. Dietary intake was assessed using 3-day food records. Ten metabolic disease-related single nucleotide polymorphisms (SNPs) were used to construct a metabolic-genetic risk score (metabolic-GRS).

Results We found significant interactions between the metabolic-GRS and total fat intake on fasting insulin level $\left(\mathrm{P}_{\text {interaction }}=0.017\right)$, insulin-glucose ratio $\left(\mathrm{P}_{\text {interaction }}=0.010\right)$ and HOMA-B $\left(\mathrm{P}_{\text {interaction }}=0.002\right)$, respectively, in addition to a borderline GRS-fat intake interaction on HOMA-IR $\left(\mathrm{P}_{\text {interaction }}=0.051\right)$. Within the high-fat intake category $[37.98 \pm 3.39 \%$ of total energy intake (TEI)], individuals with $\geq 5$ risk alleles had increased fasting insulin level $(\mathrm{P}=0.021)$, insulin-glucose ratio $(\mathrm{P}=0.010)$, HOMA-B $(\mathrm{P}=0.001)$ and HOMA-IR $(\mathrm{P}=0.053)$ than those with $<5$ risk alleles.

Conclusion Our study has demonstrated a novel GRS-fat intake interaction in young Brazilian adults, where individuals with higher genetic risk and fat intake had increased glucose and insulin-related traits than those with lower genetic risk. Large intervention and follow-up studies with an objective assessment of dietary factors are needed to confirm our findings.
\end{abstract}

Keywords Genetic risk score $\cdot$ Metabolic traits $\cdot$ Brazil $\cdot$ Fat intake $\cdot$ Gene-diet interaction

Maria Aderuza Horst, Karani Santhanakrishnan Vimaleswaran have contributed equally to this work.

Maria Aderuza Horst

aderuza@ufg.br

$\triangle$ Karani Santhanakrishnan Vimaleswaran

v.karani@ reading.ac.uk

1 Department of Food and Nutritional Sciences, Hugh Sinclair Unit of Human Nutrition, University of Reading, Reading RG6 6DZ, UK

2 Department of Clinical Nutrition, Faculty of Applied Medical Sciences, King Abdulaziz University, Jeddah, Saudi Arabia

3 Nutritional Genomics Research Group, Faculty of Nutrition, Federal University of Goiás (UFG), Goiania, Goiás, Brazil

4 Institute for Cardiovascular and Metabolic Research, University of Reading, Reading, UK

5 Institute for Food, Nutrition, and Health, University of Reading, Reading, UK

\section{Introduction}

Metabolic diseases, such as type 2 diabetes (T2D), have been recognised as a significant public health problem worldwide $[1,2]$, playing a critical role in medical impoverishment [3-6]. T2D is a major contributor to morbidity and mortality and individuals with T2D have a five-fold increased risk of developing cardiovascular diseases (CVD) [7]. The prevalence of diabetes has increased globally (over 463 million adults) [8] but at a faster rate in low- and middle-income countries (LMICs) [9]. In Brazil, the prevalence of T2D has increased by 24\% between 2006 and 2019 [10] and an estimate of 65,581 deaths have been shown to be caused by diabetes among adults aged $35-80$ years [11]. It has been reported that the prevalence of prediabetes and T2D among Brazilian adolescents were $22.0 \%$ and $3.3 \%$, respectively [12]. Studies have also demonstrated the occurrence of cardiometabolic risk factors including abdominal obesity, high 
insulin levels, dyslipidaemia, and high blood pressure among Brazilian adolescents [12-14]. Hence, early interventions targeting these factors might be an effective strategy to prevent or slow the progression of T2D and decrease the risk of CVD and associated premature mortality [8].

Much of the increase in the prevalence of metabolic diseases in Brazil is attributed to an epidemiological transition characterised by changes in Brazilian age structure, population ageing, reduced rates of infant mortality and fertility and increased low birth weight [15-19]. Changes in the cultural and socioeconomic patterns, for instance, increasing urbanisation and economic improvement, have led to negative changes in lifestyle behaviours, including physical inactivity and unhealthy diet, in the Brazilian adolescent/ young adult population [20]. A previous study has shown that the intake of saturated fatty acids (SFA) was higher in adolescents than adults in Brazil [21]. Animal and human studies have demonstrated an association between increased dietary fat intake and increased insulin resistance [22-24]. In addition, the dietary behaviours of Brazilian young adults have been shown to be characterised by higher intakes of unhealthy foods than middle-aged and older adults, highlighting the need for age-specific public health interventions [25].

The development of metabolic diseases such as T2D is closely linked to a complex interplay between genetic and lifestyle factors, such as diet. Numerous genetic loci have been shown to be associated with T2D [26-29] and related traits [30,31] and, to date, 243 genetic loci have been identified to be associated with the risk of T2D in multiple ethnic groups [26-29]. Single nucleotide polymorphisms (SNPs) have only a modest effect on disease risk, thus, generating a genetic risk score (GRS) combining several SNPs across the genome is necessary for increasing power to identify disease predisposition patterns of an individual [32]. Evidence has suggested that the genetic risk of metabolic diseases can be modified by dietary intake [33-37]. There are a few genediet interaction studies in Brazilians; however, the studies have focused only on cardiovascular disease related traits [38-40]. To date, there are no GRS-diet interaction studies on metabolic traits in Brazilians. Hence, we aimed to investigate the interaction of 10 metabolic disease-related SNPs, as a GRS, with dietary intake on metabolic traits in 200 healthy Brazilian young adults.

\section{Methodology}

\section{Study population}

Obesity, Lifestyle and Diabetes in Brazil (BOLD) is a crosssectional study of Brazilian healthy young adults aged 19-24 years recruited at the Federal University of Goiás
(UFG) between March and June 2019. This study was conducted as part of the ongoing GeNuIne (gene-nutrient interactions) Collaboration, which aims to investigate the impact of genes and lifestyle factors on chronic diseases using data from multiple ethnic groups [41, 42]. All participants completed baseline questionnaires regarding health status, demographics, and socioeconomic status. The study exclusion criteria included those who are 1) using lipid-lowering or hypoglycemic drugs and mineral or vitamin supplements, 2) undergoing dietary interventions in the last 6 months, 3 ) having acute clinical conditions such as infection, inflammation, fever or diarrhoea, or confirmed diagnosis of chronic diseases such as diabetes mellitus, moderate/severe hypertension, cancer, rheumatoid arthritis and cardiovascular complications, 4) doing vigorous physical activity. In total, 416 individuals showed interest in participating in the study. However, 207 participants met the inclusion criteria and only 200 completed the study (Fig. 1). Out of the 200 participants, only 194 had information on genetic and phenotypic measurements as DNA samples were not available for 6 participants. The study was approved by the Ethics Committee of the Federal University of Goiás (protocol number 3.007.456, 08/11/2018), and performed according to the ethical principles in the Declaration of Helsinki. All participants gave written informed consent for study participation.

\section{Anthropometric and biochemical measurements}

Body weight, height and waist circumference (WC) were measured using standardized methods. The weighing was performed on a Tanita ${ }^{\circledR}$ portable electronic scale, with a maximum capacity of $150 \mathrm{~kg}$. For height, a stadiometer with a movable rod was used. WC was measured using an inelastic measuring tape. Body mass index (BMI) was calculated as weight in kilograms divided by height in meters ${ }^{2}$ and WC measurement was taken using a non-extensible measuring tape with partici-pants in light clothing [43]. Body composition was performed by Dual Energy Radiological Absorptiometry (DXA), using the Lunar DPX NT model (General Electric Medical Systems Lunar®; Madison, USA).

Blood samples were collected by peripheral venous puncture in the morning after a 12-h fast and the volunteers were advised not to consume alcohol $72 \mathrm{~h}$ before the blood collection. Samples were immediately processed after the collection at the Romulo Rocha Laboratory (Goiânia, Brazil). Fasting serum glucose and insulin were collected in BD Vacutainer ${ }^{\circledR}$ tube and determined by the enzymatic colorimetric method, with an automatic System Vitros Chemistry 950 XRL (Johnson \& Johnson, New Brunswick, NJ, USA). Plasma glycated haemoglobin $(\mathrm{HbAlc})$ was collected in an ethylene-diamine-tetraacetic acid (EDTA) tubes BD Vacutainer ${ }^{\circledR}$ and measured using high-pressure chromatography (HPLC-Bio-Rad 


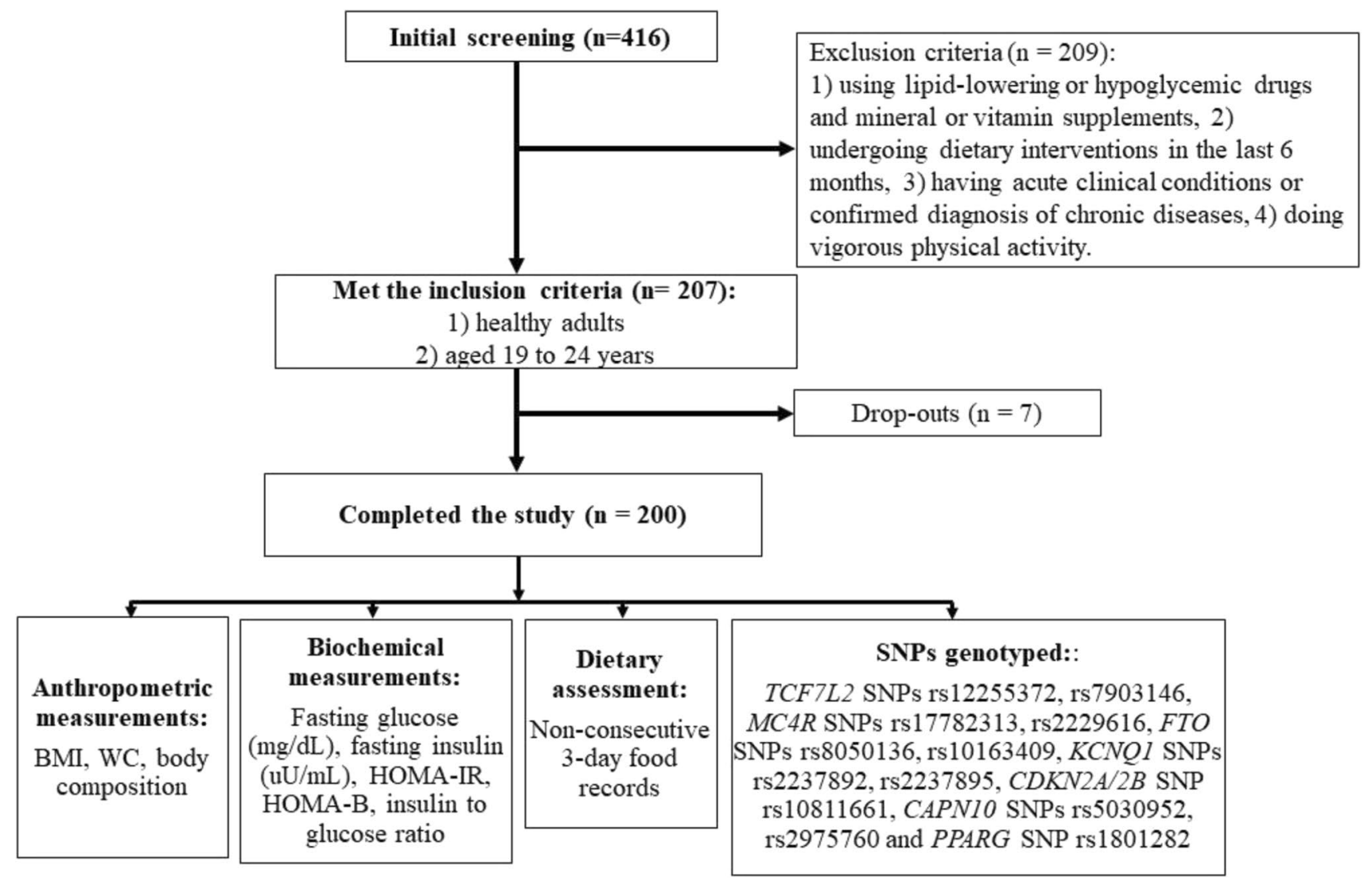

Fig. 1 Flow chart showing the participant recruitment process in the BOLD study. In total, 416 individuals were initially screened. After excluding participants based on the exclusion criteria, 207 were included in the study. However, only 200 completed the study. BMI body mass index, $W C$ waist circumference, $H b A l c$ glycated haemoglobin A1c, HOMA-IR homeostasis model assessment estimate of

Laboratories, Hercules, CA, USA). Plasma samples were obtained by centrifugation at $3500 \mathrm{rpm}$ for ten minutes at $4{ }^{\circ} \mathrm{C}$. The homeostasis model assessment (HOMA) was used to assess the degree of insulin resistance (IR) (HOMA-IR) and $\beta$-cell function (HOMA-B). HOMA-IR and HOMA-B were calculated as follows: [fasting insulin levels $(\mathrm{mU} / \mathrm{l}) \times$ fasting glucose levels $(\mathrm{mmol} / \mathrm{l}) / 22.5]$ and [20 $\times$ fasting insulin levels)/(fasting glucose levels -3.5 ], respectively [44].

\section{Dietary assessment}

Food intake was assessed by trained nutritionists using nonconsecutive 3-day food records, including a weekend day [45]. Individuals were provided with measuring cups and spoons of different sizes to assist them in estimating portion size for each food. Foods consumed were converted into grams using the Avanutri Online ${ }^{\circledR}$ diet calculation software (Avanutri Informática Ltda, Rio de Janeiro, Brazil). insulin resistance, HOMA- $B$ homeostasis model assessment estimate of insulin secretion, TCF7L2 Transcription factor 7-like 2, MC4R melanocortin 4 Receptor, $P P A R G$ Peroxisome proliferator-activated receptor gamma, FTO fat mass and obesity-associated, $C D K N 2 A / 2 B$ Cyclin dependent kinase inhibitor 2A/2B, KCNQ1 Potassium voltagegated channel subfamily Q member 1 and CAPN10 Calpain 10

\section{Genotyping}

The blood samples ( $3 \mathrm{ml}$ each) were collected in an EDTA tubes BD Vacutainer® tubes and transported at a controlled temperature $\left(-80^{\circ} \mathrm{C}\right)$ by the World Courier Company to perform genotyping at LGC Genomics (http://www.lgcgroup. com/services/genotyping), employing the competitive allelespecific PCR-KASP® assay.

\section{SNP selection and GRS calculation}

We selected 12 SNPs that have shown associations with metabolic traits in multiple ethnic groups [26-31]. The detailed information of these SNPs is shown in Table S1. Two SNPs were excluded from the current analysis, as the Calpain 10 (CAPN10) rs2975760 SNP was not in Hardy-Weinberg equilibrium (HWE) and the melanocortin 4 Receptor $(M C 4 R)$ rs2229616 SNP had a minor allele frequency (MAF) of less than 1\%. Unweighted metabolicGRS was calculated by summing the number of risk alleles 
present in each individual. The GRS was generated from the following SNPs: rs 12255372, rs7903146 of the Transcription factor 7-like 2 (TCF7L2) gene, rs17782313 of the MC4R gene, rs 8050136 and rs 10163409 of the fat mass and obesity-associated (FTO), rs2237892 and rs2237895 of the Potassium voltage-gated channel subfamily Q member 1(KCNQ1) gene, rs10811661 of the Cyclin dependent kinase inhibitor $2 \mathrm{~A} / 2 \mathrm{~B}(C D K N 2 A / 2 B)$ gene, rs5030952 of the CAPN10 gene, and rs 1801282 of the Peroxisome proliferator-activated receptor gamma (PPARG) gene. Genotypes were coded as 0,1 , or 2 according to the number of metabolic-associated risk alleles that are defined based on the literature. These values were then calculated by summing the number of risk alleles for each variant. The GRS was then categorised based on the median risk alleles into two categories: "GRS $<5$ risk alleles" and "GRS $\geq 5$ risk alleles".

\section{Statistical analysis}

Descriptive characteristics of the study population stratified by sex were presented as means and standard deviation (SDs) for continuous variables and compared using an independent samples t-test. Variables were tested for normality using Shapiro-Wilk's W test and non-normally distributed variables were log-transformed including BMI, WC, body fat mass percentage, $\mathrm{HbA1c}$, fasting glucose, fasting insulin, HOMA-IR, HOMA-B, insulin to glucose ratio, total energy intake (TEI), carbohydrate $\%$, protein $\%$, SFA $\%$, and polyunsaturated fatty acids (PUFA) $\%$. We investigated the effects of metabolic-GRS on metabolic traits using general linear models. To test the interactions of the metabolic-GRS with dietary factors on metabolic traits, we included the interaction term (e.g., GRS*fat intake) in the models. The dietary factors investigated in our study included the total dietary intake of fat, protein, and carbohydrate (percentages of TEI). Significant interactions between the GRS and the total fat intake were analysed in more depth to determine the effect of fat subtypes including SFA, monounsaturated fatty acids (MUFA), and PUFA. The GRS-nutrient interactions that reached statistical significance $(p<0.05)$ were tested for the effects of the GRSs on metabolic traits according to tertiles of dietary intakes (low, medium and high intake) using general linear models. All models were adjusted for age, sex and BMI (when BMI is not an outcome). Given that insulin levels are influenced by both the capacity for insulin secretion and IR [46, 47], analysis of HOMA-B was performed with and without adjustment for IR to improve the accuracy of pancreatic $\beta$-cell function estimate. All statistical tests were two-sided, and analyses were performed using Statistical Package for the Social Sciences (SPSS) software (version 24; SPSS Inc., Chicago, IL, USA).

\section{Results}

\section{Characteristics of the study participants}

Table 1 summarises the characteristics of individuals in this study according to sex. Men had higher BMI, WC, fasting glucose, and lower fat mass \% compared to women ( $\mathrm{P}<0.05$ for all). Men also reported higher intakes of total energy and protein than women $(\mathrm{P}<0.05$ for all $)$.

\section{Associations between metabolic-GRS and metabolic traits}

None of the associations between metabolic-GRS and metabolic-disease related traits was statistically significant except for the association with BMI $(\mathrm{P}=0.008)$ (Table 2).

\section{Interactions of metabolic-GRS with dietary factors on metabolic traits}

As shown in Table 3, there were statistically significant interactions between the metabolic-GRS and total fat intake $\left(\%\right.$ of TEI) on fasting insulin level $\left(\mathrm{P}_{\text {interaction }}=0.017\right)$, insulin-glucose ratio $\left(\mathrm{P}_{\text {interaction }}=0.010\right)$ and HOMA-B $\left(\mathrm{P}_{\text {interaction }}=0.002\right)$ and a borderline interaction on HOMAIR $\left(\mathrm{P}_{\text {interaction }}=0.051\right)$. Among those in the highest tertile of fat intake $(37.98 \pm 3.39 \%$ of TEI), individuals with $\geq 5$ risk alleles had higher fasting insulin level $(\mathrm{P}=0.021)$, insulin-glucose ratio $(\mathrm{P}=0.010)$, HOMA-B $(\mathrm{P}=0.001)$ and HOMA-IR $(\mathrm{P}=0.053)$, compared to those with $<5$ risk alleles (Figs. 2 and 3). Interaction on HOMA-B was still significant after adjusting the analysis for HOMAIR $\left(\mathrm{P}_{\text {interaction }}=0.016\right)$, Figure $\mathrm{S} 1$. We further examined interactions with fat subtypes on these traits. Significant interactions were detected between the metabolic-GRS and MUFA intake on fasting insulin $\left(\mathrm{P}_{\text {interaction }}=0.021\right)$, HOMA-IR $\left(\mathrm{P}_{\text {interaction }}=0.021\right)$ and insulin to glucose ratio $\left(\mathrm{P}_{\text {interaction }}=0.031\right)$, however, none of these interactions was statistically significant after tertile analysis. Significant interactions were also observed between the metabolic-GRS and intakes of total fat, PUFA and MUFA on percentage of body fat mass $\left(\mathrm{P}_{\text {interaction }}=0.009,0.033\right.$ and 0.038 , respectively).

\section{Discussion}

The present study investigated the potential interplay between metabolic-GRS and dietary macronutrient intake on metabolic traits in a Brazilian young adult population. Our results provide evidence of significant GRS-fat intake interactions on glucose and insulin-related traits, where 
Table 1 Characteristics of study participants

\begin{tabular}{lcccc}
\hline Parameters & Total $(\mathrm{n}=200)$ & Women $(\mathrm{n}=147)$ & Men $(\mathrm{n}=53)$ & $p$-Value \\
\hline Age (years) & $21.35 \pm 1.67$ & $21.33 \pm 1.70$ & $21.40 \pm 1.61$ & 0.815 \\
BMI (kg/m2) & $23.35 \pm 4.42$ & $22.81 \pm 3.97$ & $24.86 \pm 5.23$ & $\mathbf{0 . 0 0 4}$ \\
WC (cm) & $74.55 \pm 13.56$ & $71.10 \pm 12.05$ & $84.13 \pm 13.01$ & $\mathbf{0 . 0 0 0}$ \\
Body fat mass (\%) & $33.91 \pm 10.72$ & $37.17 \pm 8.77$ & $24.84 \pm 10.48$ & $\mathbf{0 . 0 0 0}$ \\
HbA1c (\%) & $4.73 \pm 0.25$ & $4.71 \pm 0.25$ & $4.78 \pm 0.26$ & 0.103 \\
Fasting serum glucose (mg/dL) & $87.18 \pm 6.84$ & $86.43 \pm 6.78$ & $89.26 \pm 6.60$ & $\mathbf{0 . 0 0 9}$ \\
Fasting serum insulin (uU/mL) & $8.74 \pm 3.80$ & $8.69 \pm 3.37$ & $8.88 \pm 4.82$ & 0.784 \\
HOMA-IR & $1.89 \pm 0.88$ & $1.86 \pm 0.76$ & $1.98 \pm 1.15$ & 0.513 \\
HOMA-B & $138.32 \pm 65.75$ & $142.47 \pm 65.65$ & $126.81 \pm 65.25$ & 0.137 \\
Insulin to glucose ratio & $0.10 \pm 0.04$ & $0.10 \pm 0.04$ & $0.10 \pm 0.05$ & 0.944 \\
Energy (Kcal/day) & $1827.81 \pm 597.94$ & $1741.52 \pm 558.82$ & $2067.15 \pm 641.91$ & $\mathbf{0 . 0 0 1}$ \\
Protein (energy \%) & $17.11 \pm 3.63$ & $16.74 \pm 3.33$ & $18.14 \pm 4.24$ & $\mathbf{0 . 0 1 6}$ \\
Carbohydrate (energy \%) & $51.09 \pm 7.11$ & $51.11 \pm 7.01$ & $51.05 \pm 7.44$ & 0.961 \\
Fat (energy \%) & $31.66 \pm 5.83$ & $32.12 \pm 5.69$ & $30.38 \pm 6.08$ & 0.061 \\
SFA (\%) & $9.43 \pm 5.43$ & $9.54 \pm 6.030$ & $9.14 \pm 3.25$ & 0.652 \\
PUFA (\%) & $5.13 \pm 2.27$ & $5.08 \pm 2.38$ & $5.26 \pm 1.92$ & 0.628 \\
MUFA (\%) & $7.72 \pm 2.63$ & $7.55 \pm 2.55$ & $8.19 \pm 2.79$ & 0.129 \\
\hline
\end{tabular}

Data presented as the mean \pm SDs. $P$ values for the differences in the means between men and women were calculated using the independent samples t-test. $B M I$ body mass index, $W C$ waist circumference, $H b A l c$ glycated haemoglobin, HOMA-IR homeostasis model assessment estimate of insulin resistance, HOMA-B homeostasis model assessment estimate of insulin secretion, SFA saturated fatty acids, MUFA monounsaturated fatty acids, $P U F A$ polyunsaturated fatty acids

\begin{tabular}{lccc}
\hline Parameters & GRS $<5(\mathrm{n}=93)$ & GRS $\geq 5(\mathrm{n}=101)$ & $p$-Value \\
\hline BMI $(\mathrm{kg} / \mathrm{m} 2)$ & $23.90 \pm 0.43$ & $22.60 \pm 0.43$ & $\mathbf{0 . 0 0 8}$ \\
WC $(\mathrm{cm})$ & $75.53 \pm 1.27$ & $73.93 \pm 1.26$ & 0.967 \\
Body fat mass $(\%)$ & $35.80 \pm 1.05$ & $31.91 \pm 1.10$ & 0.663 \\
HbA1c (\%) & $4.72 \pm 0.03$ & $4.73 \pm 0.03$ & 0.964 \\
Fasting glucose (mg/dL) & $87.54 \pm 0.68$ & $86.74 \pm 0.72$ & 0.419 \\
Fasting insulin (uU/mL) & $8.91 \pm 0.43$ & $8.52 \pm 0.34$ & 0.542 \\
HOMA-IR & $1.93 \pm 0.10$ & $1.84 \pm 0.08$ & 0.663 \\
HOMA-B & $138.76 \pm 7.15$ & $138.17 \pm 6.32$ & 0.234 \\
HOMA-B adjusted for HOMA-IR & $138.76 \pm 7.15$ & $138.17 \pm 6.32$ & 0.235 \\
Insulin to glucose ratio & $0.10 \pm 0.00$ & $0.10 \pm 0.00$ & 0.477 \\
\hline
\end{tabular}

Data are Mean \pm standard error of the mean (SEM). $P$ values obtained from the linear regression analysis adjusted for age, sex and additionally for BMI when BMI is not an outcome. The analysis was performed on log-transformed variables. GRS genetic risk score, $B M I$ body mass index, $W C$ waist circumference, HbAIc glycated haemoglobin, HOMA-IR homeostasis model assessment estimate of insulin resistance, HOMA-B homeostasis model assessment estimate of insulin secretion individuals with $\geq 5$ risk alleles had higher fasting insulin level, insulin-glucose ratio, HOMA-IR and HOMA-B than those with $<5$ risk alleles among those in the high fat intake group $(37.98 \pm 3.39 \%$ of TEI). These findings suggest that individuals with $\geq 5$ risk alleles are sensitive to dietary fat with respect to glucose and insulin-related traits and that these individuals may derive the most benefit from following the Brazilian dietary guidelines which aim at reducing fat intake to less than $30 \%$ of TEI [48]. This could have significant implication for public health in terms of providing early intervention to young adults with high genetic risk before the onset of disease, which might help halt the development of T2D.

In the present study, the metabolic-GRS was found to be associated with lower BMI, which contradicts the findings of the previous GRS-related studies in European populations [49-52]. However, the Brazilian population has a mixed genetic ancestry that originates from Europeans, Africans and Native Amerindians, which might explain the discrepancies between our findings and genetic association 
Table 3 Interactions of the metabolic-GRS with dietary factors on metabolic traits

\begin{tabular}{lllllll}
\hline & Protein $(\%)$ & Carbohydrate $(\%)$ & $\begin{array}{l}\text { Fat } \\
(\%)\end{array}$ & SFA $(\%)$ & $\begin{array}{l}\text { PUFA } \\
(\%)\end{array}$ & $\begin{array}{l}\text { MUFA } \\
(\%)\end{array}$ \\
\hline BMI $(\mathrm{kg} / \mathrm{m} 2)$ & 0.255 & 0.120 & 0.922 & - & - & - \\
WC $(\mathrm{cm})$ & 0.124 & 0.303 & 0.979 & - & - & - \\
Body fat mass $(\%)$ & 0.451 & 0.311 & $\mathbf{0 . 0 0 9}$ & 0.255 & $\mathbf{0 . 0 3 3}$ & $\mathbf{0 . 0 3 8}$ \\
HbA1c (\%) & 0.955 & 0.653 & 0.632 & - & - & - \\
Fasting glucose (mg/dL) & 0.764 & 0.142 & 0.099 & - & - & - \\
Fasting insulin (uU/mL) & 0.898 & 0.37 & $\mathbf{0 . 0 1 7}$ & 0.233 & 0.809 & $\mathbf{0 . 0 2 1}$ \\
HOMA-IR & 0.944 & 0.561 & $\mathbf{0 . 0 5 1}$ & 0.357 & 0.837 & $\mathbf{0 . 0 2 1}$ \\
HOMA-B & 0.797 & 0.089 & $\mathbf{0 . 0 0 2}$ & 0.079 & 0.749 & 0.123 \\
HOMA-B adjusted for HOMA-IR & 0.784 & 0.084 & $\mathbf{0 . 0 1 6}$ & 0.131 & 0.806 & 0.952 \\
Insulin to glucose ratio & 0.895 & 0.274 & $\mathbf{0 . 0 1 0}$ & 0.154 & 0.801 & $\mathbf{0 . 0 3 1}$ \\
\hline
\end{tabular}

Data are $\mathrm{P}$ values of interaction which obtained from the linear regression analysis adjusted for age, sex and additionally for BMI when BMI is not an outcome. The analysis was performed on log-transformed variables. $G R S$ genetic risk score, $B M I$ body mass index, $W C$ waist circumference, $H b A l c$ glycated haemoglobin, HOMA-IR homeostasis model assessment estimate of insulin resistance, HOMA-B homeostasis model assessment estimate of insulin secretion, SFA saturated fatty acids, MUFA monounsaturated fatty acids, PUFA polyunsaturated fatty acids studies in Europeans [53]. Furthermore, a large GWAS of 241,258 European adults showed that the risk allele T of TCF7L2 rs7903146 was associated with lower BMI compared to the non-risk allele, which may provide a possible explanation of our findings [54]. Metabolic diseases are complex and multifactorial influenced by both environmental and genetic factors including dozens or even hundreds of genetic variants each contributing small effects on these traits $[55,56]$. Thus, the effect of unmeasured factors on BMI might influence the observed findings.

The present study found that, within the high-fat intake category, individuals with higher metabolic-GRS showed increased fasting insulin level, insulin-glucose ratio, HOMA-IR and HOMA-B, whereas those with lower GRS showed a reduction in these traits. Although direct comparison of our study with previous gene-diet interaction studies is difficult due to differences in the methodology related to the construction of GRSs and measurement of dietary intake, sample size, study design, and ethnicity, our findings are in agreement with some of the previous studies in other populations in which fat intake was found to interact with GRS on metabolic traits [33-35]. In a recent study in 302 Ghanaian adults, a GRS of 4 metabolic-related variants was associated with higher WC among individuals with high fat intake $(34.99 \pm 5.54 \%$ TEI) [57]. Data from an intervention study in 733 European adults also reported that higher total fat intake was associated with increased fasting glucose in individuals with higher GRS of 14 fasting glucose-associated SNPs and with decreased fasting glucose among individuals with lower GRS [33]. Taken together, these observations suggest that individuals with higher genetic risk might benefit more from reducing fat intake in terms of lowering their metabolic risk.

Dietary guidelines have recommended to limit the dietary intake of total fat (between 15 and 30\% of TEI) to preserve overall health and reduce the risk of developing metabolic diseases [58]. Previous studies have demonstrated that the higher intake of total fat contributes to the development of T2D by inducing IR [24, 59]. Lowering total fat intake have been reported to improve glycemic control in a systematic review of clinical trials of diabetic individuals [60]. Evidence from two previous intervention studies including individuals from various ethnic groups $(\mathrm{n}=3,234$ and 522, respectively) and with long follow-up (2.8 and 3.2 years, respectively) have also shown that decreasing fat intake (from $6.6 \pm 0.2 \%$ of TEI and to $<30 \%$ of TEI, respectively) is effective in reducing the incidence of T2D by up to $58 \%$ [61, 62]. In addition, dietary intervention in 48,835 postmenopausal women from the US showed that reducing total fat intake (by $\sim 8 \%$ of TEI) and increasing carbohydrate intake (by $~ 8 \%$ of TEI) through increasing intake of vegetable/fruit (five servings per day) and grain (six servings per day) were associated with a reduction in glycemia and diabetes progression [63]. The dietary intake of Brazilians is characterised by unfavourable fat profile with high intakes of SFA and trans fatty acids and imbalances in the omega6:omega-3 ratio, being compatible with a high risk of metabolic diseases [21]. In our study, the mean fat intake of the total sample $(31.66 \pm 5.8 \%$ of TEI) and the high fat intake group ( $37.98 \pm 3.39 \%$ of TEI) were above the recommended dietary guidelines for Brazilian adults ( $<30 \%$ of TEI) [64].

The mechanisms by which dietary fat influences IR and $\beta$-cell function are unclear; however, several pathways are 


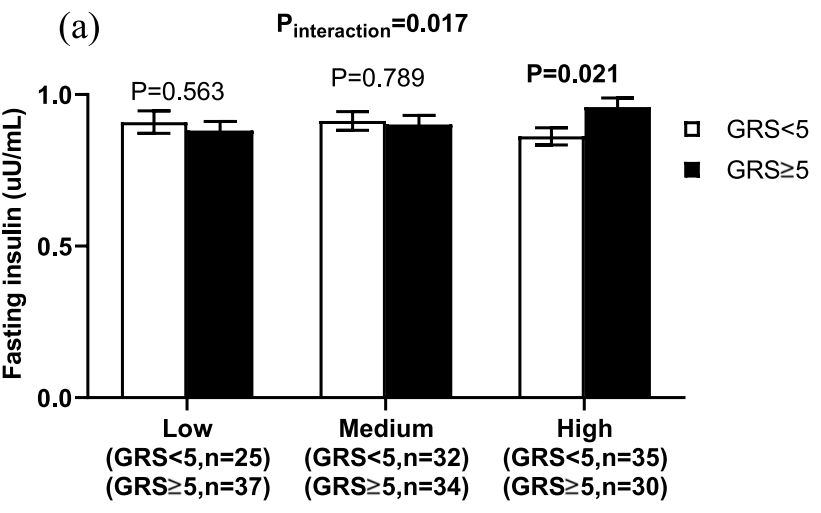

Fat intake (\%)

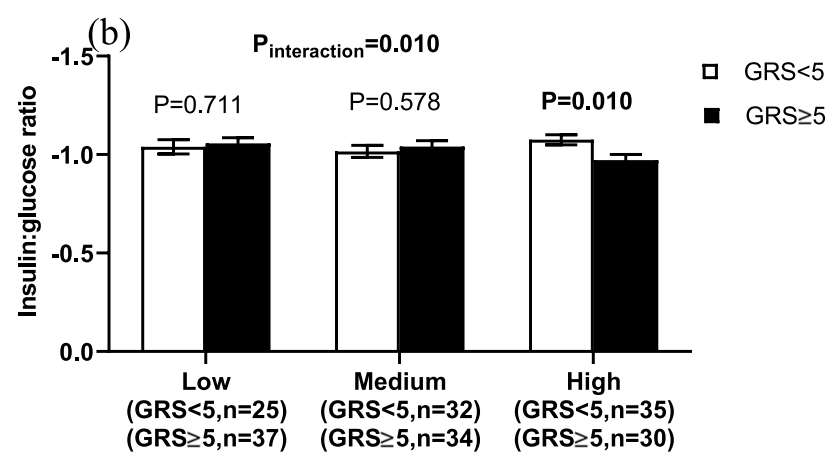

Fat intake (\%)

Fig. 2 Interaction between the metabolic-GRS and fat intake (\%) on fasting insulin levels and insulin: glucose ratio. White bars indicate individuals with GRS $<5$ risk alleles; the black bars indicate individuals with GRS $\geq 5$ risk alleles; Error bars indicate the standard error of the mean. Individuals with $\geq 5$ risk alleles had higher fasting insulin (a) and insulin to glucose ratio (b) compared to those with $<5$ risk alleles, among individuals with a higher total fat intake $(37.98 \pm 3.39 \%$ of TEI). GRS genetic risk score, TEI total energy intake

biologically plausible. IR is often mediated by increased inflammation that has been shown to be induced mostly by the effect of the fatty acids composition of the diet [65]. In particular, SFA and omega-6 have pro-inflammatory effects, and omega-3 fatty acids have anti-inflammatory effects [65]. Some of the molecular mechanisms of IR include the lipidoverload hypothesis in which ceramides or diacylglycerides are accumulated leading to the inhibition of insulin signalling and oxidative stress induced by excessive generation of free radicals or endoplasmic reticulum stress induced by excessive calorie intake [66-68]. In addition to the insulinrelated traits, there was also a significant interaction between GRS and intakes of total fat, PUFA and MUFA on the percentage of body fat mass in our study. Given that adipose tissue is a central metabolic organ that stores excess fat energy in the form of lipid and secretes proinflammatory adipokines that can also influence signalling of insulin, our finding is (a)

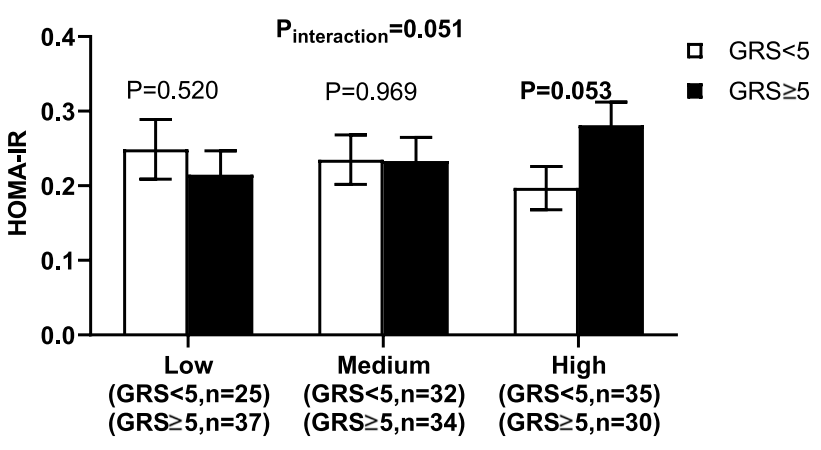

Fat intake (\%)

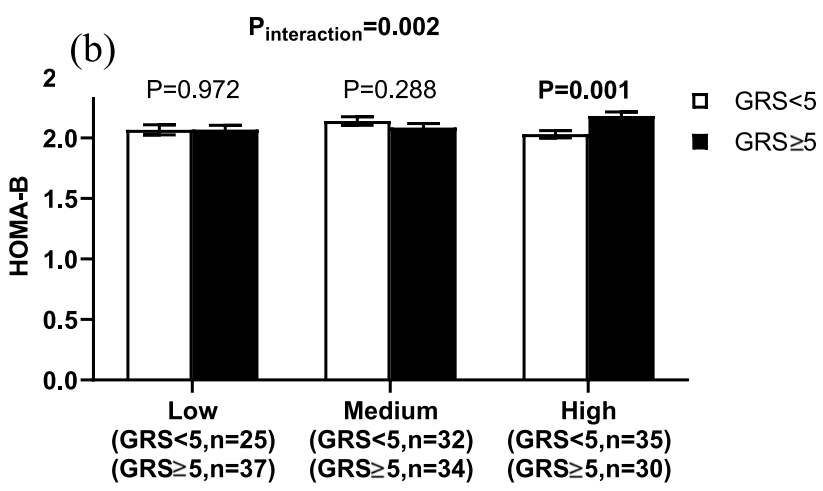

Fat intake (\%)

Fig. 3 Interaction between the metabolic-GRS and fat intake (\%) on HOMA-IR and HOMA-B. White bars indicate individuals with GRS $<5$ risk alleles; the black bars indicate individuals with GRS $\geq 5$ risk alleles; Error bars indicate the standard error of the mean. Individuals with $\geq 5$ risk alleles had higher HOMA-IR (a) and HOMA-B (b) compared to those with $<5$ risk alleles, among individuals with a higher total fat intake ( $37.98 \pm 3.39 \%$ of TEI). GRS genetic risk score, TEI total energy intake, HOMA-IR homeostasis model assessment estimate of insulin resistance, HOMA-B homeostasis model assessment estimate of insulin secretion

biologically plausible [69]. It is worth observing the intake of SFA, PUFA and MUFA which were significantly higher in the high fat intake category than low and medium intake groups; this might be one of the reasons for the observed interactions with total dietary fat intake. Evidence suggests that different types of dietary fat have differential effects on IR and insulin secretion. While a cross-sectional study in 538 Spanish individuals suggested that the intake of a MUFA-rich diet was associated with increased HOMA-B [70], a meta-analysis of randomised controlled feeding trials $(n=4220)$ demonstrated that PUFA intake showed the most consistent favourable effects in relation to improved glycaemia and insulin secretion capacity [71].

Several strengths are worth consideration. This study is the first to examine whether dietary factors interact with metabolic-GRSs on metabolic traits among the Brazilian 
young adult population. Early prediction of insulin sensitivity in young adults and effective intervention can be a critical factor in terms of delaying or preventing diabetes in normoglycemic individuals who are at risk of diabetes [72]. Also, a GRS analysis approach was used, which has the advantage over single-locus approach [32]. This approach is especially important for highly polygenic metabolic traits and can identify individuals at risk of metabolic diseases who might benefit from targeted interventions [32]. Furthermore, the study outcomes (metabolic traits) were measured using validated methods by trained staff which improve the accuracy of these estimates. Nevertheless, some limitations need to be acknowledged. A major limitation is the small sample size, suggesting that our analysis might be underpowered. However, the use of the GRS approach is suggested to improve the power and significant gene-diet interactions were detected in our study. As with all observational studies, causality between exposure and outcome cannot be inferred and residual confounders might have existed. Given the longitudinal dimension of the development of T2D and the complexity of gene-diet interactions, our cross-sectional study design fails to determine the temporality of the observed findings. Given that dietary intake was assessed using self-reported measures, we cannot exclude the effect of measurement bias. Another limitation is that the effect of different dietary sources of fat (including meat, dairy and plant) were not considered in the present analysis, which might have provided further explanations to our GRS-fat intake interactions [73]. In addition, our GRS was constructed based on 10 SNPs, which account for only a small proportion of the metabolic disease-related genetic variants. As HOMA is a widely validated clinical and epidemiological tool for assessing IR and $\beta$-cell function [74], like many other epidemiological studies [33, 35, 59], we also used HOMA-IR and HOMA-B as proxies for IR and insulin secretion, respectively. However, these measures are calculated only using fasting insulin and glucose values which might provide different estimates compared to methods based on dynamic measurements of insulin and glucose responses or those derived from clamp experiments [75]. Finally, given that the study was performed with relatively healthy overweight/obese young individuals with normal glucose tolerance who might have a quicker adaptation to changes in fat intake, the findings might not be applicable to those with impaired glucose metabolism or diabetes.

In conclusion, our study provides evidence of interactions between genetic predisposition and high fat intake on diabetes-related traits among Brazilian young adults. These findings encourage identifying Brazilian young adults with high genetic risk and tailoring dietary recommendations of fat intake according to their metabolic genetic risk profile for the primary prevention of adultonset T2D. In addition, devising polygenic risk score could be used to provide more insights on understanding the pathophysiology of the genetics of diabetes. However, large interventional and follow up studies with a more comprehensive and objective assessment of environmental factors are needed in Brazilians to confirm our findings and to evaluate the clinical benefit of implementing precision dietary interventions based on an individual's underlying genetic risk of metabolic diseases.

Supplementary Information The online version contains supplementary material available at https://doi.org/10.1007/s40200-021-00863-7

Acknowledgements We thank all the participants from the BOLD study for their cooperation. Karani S Vimaleswaran acknowledges support from the Ministry of Higher Education of Saudi Arabia for the scholarship given to Sooad Alsulami.

Author Contributions Conceptualization, K.S.V and M.A.H.; Methodology, K.S.V., M.A.H, and S.A.; Data Collection, N.T.C., N.R.S. and A.C.A.; Software, S.A.; Validation, M.A.H., K.S.V. and S.A.; Formal Analysis, M.A.H. and S.A.; Investigation, K.S.V and M.A.H..; Resources, M.A.H and K.S.V.; Data Curation, K.S.V and M.A.H.; Writing - Original Draft Preparation, S.A. and K.S.V.; Writing Review \& Editing, K.S.V. and S.A.; Supervision, K.S.V., M.A.H. and J.A.L.; Project Administration, K.S.V. and M.A.H.; Funding Acquisition, K.S.V. and M.A.H. All authors have read, edited, and approved the published version of the manuscript. All authors have read and agreed to the published version of the manuscript.

Funding The study was funded by the Conselho Nacional das Fundações Estaduais de Amparo à Pesquisa (CONFAP)-UK Academies Researcher Mobility award.

Data availability The data that support the findings of this study are available from the corresponding author (KSV) upon reasonable request.

Code availability Not applicable.

\section{Declarations}

Conflict of interest The authors declare no conflict of interest.

Ethical approval The study was conducted according to the guidelines of the Declaration of Helsinki and approved by the Ethics Committee of the Federal University of Goiás (protocol number 3.007.456, 08/11/2018).

Consent to participate All participants gave written informed consent for study participation.

Consent for publication All participants gave written informed consent for the publication of study findings.

Open Access This article is licensed under a Creative Commons Attribution 4.0 International License, which permits use, sharing, adaptation, distribution and reproduction in any medium or format, as long as you give appropriate credit to the original author(s) and the source, provide a link to the Creative Commons licence, and indicate if changes were made. The images or other third party material in this article are included in the article's Creative Commons licence, unless indicated 
otherwise in a credit line to the material. If material is not included in the article's Creative Commons licence and your intended use is not permitted by statutory regulation or exceeds the permitted use, you will need to obtain permission directly from the copyright holder. To view a copy of this licence, visit http://creativecommons.org/licenses/by/4.0/.

\section{References}

1. Horton R. Offline: Time to radically rethink non-communicable diseases. Lancet (London, England). 2019;393(10184):1922.

2. Nugent R, Fottrell E. Non-communicable diseases and climate change: linked global emergencies. Lancet (London, England). 2019;394(10199):622-3.

3. Ghebreyesus TA. Acting on NCDs: counting the cost. Lancet (London, England). 2018;391(10134):1973-4.

4. Jaspers L, Colpani V, Chaker L, van der Lee SJ, Muka T, Imo $\mathrm{D}$, et al. The global impact of non-communicable diseases on households and impoverishment: a systematic review. Eur J Epidemiol. 2015;30(3):163-88.

5. Niessen LW, Mohan D, Akuoku JK, Mirelman AJ, Ahmed S, Koehlmoos TP, et al. Tackling socioeconomic inequalities and non-communicable diseases in low-income and middle-income countries under the Sustainable Development agenda. Lancet (London, England). 2018;391(10134):2036-46.

6. Nugent R, Bertram MY, Jan S, Niessen LW, Sassi F, Jamison DT, et al. Investing in non-communicable disease prevention and management to advance the Sustainable Development Goals. Lancet (London, England). 2018;391(10134):2029-35.

7. WHO. Diet, Nutrition and the Prevention of Chronic Diseases. 2003 [Available from: https://www.who.int/dietphysicalactivity/ publications/trs916/en/.

8. IDF. Diabetes Atlas 9th edition 2019 [Available from: https:// www.diabetesatlas.org/en/.

9. Zhou B, Lu Y, Hajifathalian K, Bentham J, Cesare MD, Danaei $\mathrm{G}$, et al. Worldwide trends in diabetes since 1980: a pooled analysis of 751 population-based studies with 4.4 million participants. Lancet (London, England). 2016;387(10027):1513-30.

10. Cohen RV, Drager LF, Petry TBZ, Santos RD. Metabolic health in Brazil: trends and challenges. Lancet Diabetes Endocrinol. 2020;8(12):937-8.

11. Bracco PA, Gregg EW, Rolka DB, Schmidt MI, Barreto SM, Lotufo PA, et al. A nationwide analysis of the excess death attributable to diabetes in Brazil. Journal of global health. 2020;10(1):010401.

12. Telo GH, Cureau FV, Szklo M, Bloch KV, Schaan BD. Prevalence of type 2 diabetes among adolescents in Brazil: Findings from Study of Cardiovascular Risk in Adolescents (ERICA). Pediatr Diabetes. 2019;20(4):389-96.

13. Bloch KV, Klein CH, Szklo M, Kuschnir MC, Abreu Gde A, Barufaldi LA, et al. ERICA: prevalences of hypertension and obesity in Brazilian adolescents. Revista de saude publica. 2016;50 Suppl 1(Suppl 1):9s.

14. Kuschnir MC, Bloch KV, Szklo M, Klein CH, Barufaldi LA, Abreu Gde A, et al. ERICA: prevalence of metabolic syndrome in Brazilian adolescents. Revista de saude publica. 2016;50 Suppl 1(Suppl 1):11s.

15. Goldani MZ, Mosca PRF, Portella AK, Silveira PP, Silva CHd. The impact demographic and epidemiological transition in the health of children and adolescents in Brazil. Revista HCPA. 2012;32(1):49-57.

16. Yusuf S, Reddy S, Ounpuu S, Anand S. Global burden of cardiovascular diseases: part I: general considerations, the epidemiologic transition, risk factors, and impact of urbanization. Circulation. 2001;104(22):2746-53.

17. Magno de Carvalho JA, Rodríguez-Wong LL. The transition of the age structure of the Brazilian population in the first half of the 21 st century. Saúde Pública.24(3).

18. de Souza Buriol VC, Hirakata V, Goldani MZ, da Silva CH. Temporal evolution of the risk factors associated with low birth weight rates in Brazilian capitals (1996-2011). Popul Health Metrics. 2016;14:15.

19. Travassos GF, Coelho AB, Arends-Kuenning MP. The elderly in Brazil: demographic transition, profile, and socioeconomic condition. Revista Brasileira de Estudos Populacionais. 2020;37:1-27.

20. Ventura Barbosa Gonçalves H, Canella DS, Bandoni DH. Temporal variation in food consumption of Brazilian adolescents (2009-2015). PloS one. 2020;15(9):e0239217.

21. Souza RA, Yokoo EM, Sichieri R, Pereira RA. Energy and macronutrient intakes in Brazil: results of the first nationwide individual dietary survey. Public Health Nutr. 2015;18(17):3086-95.

22. Storlien LH, Baur LA, Kriketos AD, Pan DA, Cooney GJ, Jenkins $\mathrm{AB}$, et al. Dietary fats and insulin action. Diabetologia. 1996;39(6):621-31.

23. Costacou T, Mayer-Davis EJ. Nutrition and prevention of type 2 diabetes. Annu Rev Nutr. 2003;23:147-70.

24. Lovejoy JC. The influence of dietary fat on insulin resistance. Curr DiabRep. 2002;2(5):435-40.

25. Nogueira Bezerra I, de Carvalho Gurgel AO, Bastos Barbosa RG, Bezerra da Silva Junior G. Dietary Behaviors among Young and Older Adults in Brazil. The journal of nutrition, health \& aging. 2018;22(5):575-80.

26. Fuchsberger C, Flannick J, Teslovich TM, Mahajan A, Agarwala $\mathrm{V}$, Gaulton KJ, et al. The genetic architecture of type 2 diabetes. Nature. 2016;536(7614):41-7.

27. Morris AP, Voight BF, Teslovich TM, Ferreira T, Segrè AV, Steinthorsdottir V, et al. Large-scale association analysis provides insights into the genetic architecture and pathophysiology of type 2 diabetes. Nat Genet. 2012;44(9):981-90.

28. Scott RA, Scott LJ, Mägi R, Marullo L, Gaulton KJ, Kaakinen M, et al. An Expanded Genome-Wide Association Study of Type 2 Diabetes in Europeans. Diabetes. 2017;66(11):2888-902.

29. Mahajan A, Taliun D, Thurner M, Robertson NR, Torres JM, Rayner NW, et al. Fine-mapping type 2 diabetes loci to singlevariant resolution using high-density imputation and islet-specific epigenome maps. Nat Genet. 2018;50(11):1505-13.

30. Dupuis J, Langenberg C, Prokopenko I, Saxena R, Soranzo N, Jackson AU, et al. New genetic loci implicated in fasting glucose homeostasis and their impact on type 2 diabetes risk. Nat Genet. 2010;42(2):105-16.

31. Scott RA, Lagou V, Welch RP, Wheeler E, Montasser ME, Luan J, et al. Large-scale association analyses identify new loci influencing glycemic traits and provide insight into the underlying biological pathways. Nat Genet. 2012;44(9):991-1005.

32. Udler MS, McCarthy MI, Florez JC, Mahajan A. Genetic risk scores for diabetes diagnosis and precision medicine. Endocr Rev. 2019;40(6):1500-20.

33. Wang T, Huang T, Zheng Y, Rood J, Bray GA, Sacks FM, et al. Genetic variation of fasting glucose and changes in glycemia in response to 2-year weight-loss diet intervention: the POUNDS LOST trial. International journal of obesity (2005). 2016;40(7):1164-9.

34. Eriksen R, Gibson R, Aresu M, Heard A, Chan Q, Evangelou E, et al. Gene-diet quality interactions on haemoglobin A1c and type 2 diabetes risk: The Airwave Health Monitoring Study. Endocrinology, diabetes \& metabolism. 2019;2(4):e00074.

35. Kim DS, Kim BC, Daily JW, Park S. High genetic risk scores for impaired insulin secretory capacity doubles the risk for type 
2 diabetes in Asians and is exacerbated by Western-type diets. Diabetes Metab Res Rev. 2018;34(1):e2944.

36. Qi L, Cornelis MC, Zhang C, van Dam RM, Hu FB. Genetic predisposition, Western dietary pattern, and the risk of type 2 diabetes in men. Am J Clin Nutr. 2009;89(5):1453-8.

37. Zheng JS, Li K, Huang T, Chen Y, Xie H, Xu D, et al. Genetic Risk Score of Nine Type 2 Diabetes Risk Variants that Interact with Erythrocyte Phospholipid Alpha-Linolenic Acid for Type 2 Diabetes in Chinese Hans: A Case-Control Study. Nutrients. 2017;9(4).

38. Fujii TMM, Norde MM, Fisberg RM, Marchioni DML, Rogero MM. Lipid metabolism genetic risk score interacts with the Brazilian Healthy Eating Index Revised and its components to influence the odds for dyslipidemia in a cross-sectional population-based survey in Brazil. Nutr Health. 2019;25(2):119-26.

39. Miranda AM, Steluti J, Norde MM, Fisberg RM, Marchioni DM. The association between genetic risk score and blood pressure is modified by coffee consumption: Gene-diet interaction analysis in a population-based study. Clinical nutrition (Edinburgh, Scotland). 2019;38(4):1721-8.

40. Surendran S, Morais C, Abdalla D, Shatwan I, et al. The influence of one-carbon metabolism gene polymorphisms and geneenvironment interactions on homocysteine, Vitamin B12, folate and lipids in a Brazilian adolescent population. Journal of Diabetology. 2019;10(3):110-22.

41. Vimaleswaran KS. Gene-nutrient interactions on metabolic diseases: findings from the GeNuIne collaboration. Nutr Bull. 2017;42(1):80-6.

42. Vimaleswaran KS. A nutrigenetics approach to study the impact of genetic and lifestyle factors on cardiometabolic traits in various ethnic groups: findings from the GeNuIne Collaboration. Proc Nutr Soc. 2020;79(2):194-204.

43. World Health Organization. Waist circumference and waisthip ratio Report of a WHO expert consultation 2011 [Available from: https://www.who.int/nutrition/publications/obesity/ WHO_report_waistcircumference_and_waisthip_ratio/en/.

44. Wallace TM, Levy JC, Matthews DR. Use and abuse of HOMA modeling. Diabetes Care. 2004;27(6):1487-95.

45. Yang YJ, Kim MK, Hwang SH, Ahn Y, Shim JE, Kim DH. Relative validities of 3-day food records and the food frequency questionnaire. Nurs Res Pract. 2010;4(2):142-8.

46. Bergman RN, Phillips LS, Cobelli C. Physiologic evaluation of factors controlling glucose tolerance in man: measurement of insulin sensitivity and beta-cell glucose sensitivity from the response to intravenous glucose. J Clin Investig. 1981;68(6):1456-67.

47. Kahn SE, Prigeon RL, McCulloch DK, Boyko EJ, Bergman RN, Schwartz MW, et al. Quantification of the relationship between insulin sensitivity and beta-cell function in human subjects. Evidence for a hyperbolic function Diabetes. 1993;42(11):1663-72.

48. Sichieri R, Chiuve SE, Pereira RA, Lopes AC, Willett WC. Dietary recommendations: comparing dietary guidelines from Brazil and the United States. Cad Saude Publica. 2010;26(11):2050-8.

49. Belsky DW, Moffitt TE, Sugden K, Williams B, Houts R, McCarthy J, et al. Development and evaluation of a genetic risk score for obesity. Biodemography Soc Biol. 2013;59(1):85-100.

50. Li S, Zhao JH, Luan J, Luben RN, Rodwell SA, Khaw KT, et al. Cumulative effects and predictive value of common obesitysusceptibility variants identified by genome-wide association studies. Am J Clin Nutr. 2010;91(1):184-90.

51. Peterson RE, Maes HH, Holmans P, Sanders AR, Levinson DF, Shi J, et al. Genetic risk sum score comprised of common polygenic variation is associated with body mass index. Hum Genet. 2011;129(2):221-30.

52. Sandholt CH, Sparso T, Grarup N, Albrechtsen A, Almind K, Hansen L, et al. Combined analyses of 20 common obesity susceptibility variants. Diabetes. 2010;59(7):1667-73.

53. Kehdy FS, Gouveia MH, Machado M, Magalhães WC, Horimoto AR, Horta BL, et al. Origin and dynamics of admixture in Brazilians and its effect on the pattern of deleterious mutations. Proc Natl Acad Sci USA. 2015;112(28):8696-701.

54. Justice AE, Winkler TW, Feitosa MF, Graff M, Fisher VA, Young K, et al. Genome-wide meta-analysis of 241,258 adults accounting for smoking behaviour identifies novel loci for obesity traits. Nat Commun. 2017;8:14977.

55. Franks PW, McCarthy MI. Exposing the exposures responsible for type 2 diabetes and obesity. Science (New York, NY). 2016;354(6308):69-73.

56. Locke AE, Kahali B, Berndt SI, Justice AE, Pers TH, Day FR, et al. Genetic studies of body mass index yield new insights for obesity biology. Nature. 2015;518(7538):197-206.

57. Alsulami S, Nyakotey DA, Dudek K, Bawah AM, Lovegrove JA, Annan RA, et al. Interaction between metabolic genetic risk score and dietary fatty acid intake on central obesity in a ghanaian population. Nutrients. 2020;12(7).

58. WHO. Healthy diet 2020 Available from: https://www.who.int/ news-room/fact-sheets/detail/healthy-diet.

59. Vizzuso S, Amatruda M, Del Torto A, D'Auria E, Ippolito G, Zuccotti GV, et al. Is Macronutrients Intake a Challenge for Cardiometabolic Risk in Obese Adolescents? Nutrients. 2020;12(6).

60. Wheeler ML, Dunbar SA, Jaacks LM, Karmally W, Mayer-Davis EJ, Wylie-Rosett J, et al. Macronutrients, food groups, and eating patterns in the management of diabetes: a systematic review of the literature, 2010. Diabetes Care. 2012;35(2):434-45.

61. Knowler WC, Barrett-Connor E, Fowler SE, Hamman RF, Lachin JM, Walker EA, et al. Reduction in the incidence of type 2 diabetes with lifestyle intervention or metformin. $\mathrm{N}$ Engl J Med. 2002;346(6):393-403.

62. Tuomilehto J, Lindström J, Eriksson JG, Valle TT, Hämäläinen $\mathrm{H}$, Ilanne-Parikka P, et al. Prevention of type 2 diabetes mellitus by changes in lifestyle among subjects with impaired glucose tolerance. N Engl J Med. 2001;344(18):1343-50.

63. Howard BV, Aragaki AK, Tinker LF, Allison M, Hingle MD, Johnson KC, et al. A low-fat dietary pattern and diabetes: a secondary analysis from the women's health initiative dietary modification trial. Diabetes Care. 2018;41(4):680-7.

64. Oliveira M, Santos L. Dietary guidelines for Brazilian population: an analysis from the cultural and social dimensions of food. Ciencia \& saude coletiva. 2020;25(7):2519-28.

65. Rice Bradley BH. Dietary fat and risk for type 2 diabetes: a review of recent research. Current nutrition reports. 2018;7(4):214-26.

66. Glass CK, Olefsky JM. Inflammation and lipid signaling in the etiology of insulin resistance. Cell Metab. 2012;15(5):635-45.

67. Taubes G. Insulin resistance. Prosperity's plague. Science (New York, NY). 2009;325(5938):256-60.

68. Ussher JR, Koves TR, Cadete VJ, Zhang L, Jaswal JS, Swyrd $\mathrm{SJ}$, et al. Inhibition of de novo ceramide synthesis reverses dietinduced insulin resistance and enhances whole-body oxygen consumption. Diabetes. 2010;59(10):2453-64.

69. Stienstra R, Duval C, Müller M, Kersten S. PPARs, Obesity, and Inflammation. PPAR Res. 2007;2007:95974.

70. Rojo-Martínez G, Esteva I, Ruiz de Adana MS, García-Almeida JM, Tinahones F, Cardona F, et al. Dietary fatty acids and 
insulin secretion a population-based study. European Journal of Clinical Nutrition. 2006;60(10):1195-200.

71. Imamura F, Micha R, Wu JH, de Oliveira Otto MC, Otite FO, Abioye AI, et al. Effects of Saturated Fat, Polyunsaturated Fat, Monounsaturated Fat, and Carbohydrate on GlucoseInsulin Homeostasis: A Systematic Review and Meta-analysis of Randomised Controlled Feeding Trials. PLoS Med. 2016;13(7):e1002087.

72. Penn L, Rodrigues A, Haste A, Marques MM, Budig K, Sainsbury K, et al. NHS Diabetes Prevention Programme in England: formative evaluation of the programme in early phase implementation. BMJ Open. 2018;8(2):e019467.

73. Wanders AJ, Alssema M, de Koning EJ, le Cessie S, de Vries $\mathrm{JH}$, Zock PL, et al. Fatty acid intake and its dietary sources in relation with markers of type 2 diabetes risk: The NEO study. Eur J Clin Nutr. 2017;71(2):245-51.
74. Wang C, Liu Z, Zhang P, Ma X, Che K, Wang Y. The differences in homeostasis model assessment values in type 2 diabetic patients with different lengths of history of diabetes. Archives of endocrinology and metabolism. 2019;63(3):222-7.

75. Gutch M, Kumar S, Razi SM, Gupta KK, Gupta A. Assessment of insulin sensitivity/resistance. Indian journal of endocrinology and metabolism. 2015;19(1):160-4.

Publisher's Note Springer Nature remains neutral with regard to jurisdictional claims in published maps and institutional affiliations. 